\title{
ON THE GENERIC EXISTENCE OF SPECIAL ULTRAFILTERS
}

\author{
R. MICHAEL CANJAR
}

(Communicated by Andreas R. Blass)

\begin{abstract}
We introduce the concept of the generic existence of $P$-point, $Q$ point, and selective ultrafilters, a concept which is somewhat stronger than the existence of these sorts of ultrafilters. We show that selective ultrafilters exist generically iff semiselectives do iff $m_{c}=c$, and we show that $Q$-point ultrafilters exist generically iff semi- $Q$-points do iff $m_{c}=d$, where $d$ is the minimal cardinality of a dominating family of functions and $m_{c}$ is the minimal cardinality of a cover of the real line by nowhere-dense sets. These results complement a result of Ketonen, that $P$-points exist generically iff $c=d$, and one of P. Nyikos and D. H. Fremlin, that saturated ultrafilters exist generically iff $m_{c}=c=2^{<c}$.
\end{abstract}

All our filters (and ultrafilters) are proper, nonprincipal, and on $\omega$. By the character of a filter we mean the minimum cardinality of a generating set for the filter. We use the term function exclusively for elements of ${ }^{\omega} \omega$. We say that a function $f$ dominates a function $g$ iff $\forall n \in \omega \quad f(n)>g(n)$. Following [21] we let $d$ denote the minimum cardinality of a dominating family of functions. We let $c$ be the cardinality ${ }^{\omega} \omega$ and $m_{c}$ be the minimum cardinality of a cover of the real line consisting of nowhere-dense sets. The notation $m_{c}$ comes from "Martin's Axiom for countable partial orders"; $m_{c}$ is the maximum cardinal $\lambda$ such that the following holds: given any countable partial order $P$ and fewer than $\lambda$ dense subsets of $P$, there exists a generic $G \subseteq P$ which meets each of the dense subsets. Also, $m_{c}$ may be characterized as the maximum cardinal $\lambda$ such that, given any family of functions $H$ of size $<\lambda$, we may find a function $g$ such that $\forall h \in H, \exists n \in \omega g(n)=h(n)$. Some of the basic properties of this cardinal, including proofs of the equivalence of these characterizations, may be found in [1], [15], and [22]. It is clear from the above discussion that $m_{c} \leq d \leq c$.

In this paper we will consider the question of the existence of various special sorts of ultrafilters, whose definitions we now mention. An ultrafilter $U$ is called a $P$-point if every function is either finite-to-one or bounded on a set in $U$. An ultrafilter $U$ is called a $Q$-point or rare ultrafilter if every finite-to-one function is one-to-one on a set in $U$. An ultrafilter $U$ is said to be a semi- $Q$-point (or

Received by the editors July 2, 1988 and, in revised form, May 1, 1989.

1980 Mathematics Subject Classification (1985 Revision). Primary 03E05; Secondary 03E35, $54 \mathrm{H} 05$. 
rapid) if for each finite-to-one function $f$ there exists a set $E \in U$ such that $\left|E \cap f^{-1}\{n\}\right| \leq n$ for every $n \in \omega$. (This is equivalent to the following: for each function $f$ there exists a set $E \in U$ such that the enumerating function of $E$ dominates $f$, whence the term rapid). An ultrafilter is said to be selective if it is both a $P$-point and a $Q$-point, so that every function is either one-to-one or bounded on a set in the ultrafilter. An ultrafilter is said to be semiselective iff it is both a $P$-point and a semi- $Q$-point. All of these sorts of ultrafilters are known to exist under various set-theoretic hypotheses. However it is consistent that there do not exist $P$-points [18] and it is also consistent that there do not exist $Q$-points or even semi- $Q$-points [14].

There is another characterization of $Q$-points, discovered independently by Mathias [13] and Taylor [20], which we will find useful. If $A$ is a subset of $\omega$ and $f$ is a function, then we will say that $A$ is $f$-rare if whenever $a, b \in A$ and $a<b$, then we also have $f(a)<b$. Then an ultrafilter $U$ is a $Q$-point iff $U$ contains, for each function $f$, a set which is $f$-rare. Of course in this characterization we may confine our attention to functions $f$ which are chosen from a dominating family.

We will mention related work on saturated ultrafilters, whose definition we will shortly give. First we call a filter on $\omega \times \omega$ a type. We say that a type $T$ is consistent over an ultrafilter $U$ if for every $R \in T,\{n \mid \exists m\langle n, m\rangle \in$ $R\} \in U . T$ is realized over $U$ if there exists a function $g$ such that for every $R \in T,\{n \mid\langle n, g(n)\rangle \in R\} \in U$. An ultrafilter $U$ is saturated if every type $T$ which is consistent over $U$ is also realized over $U$. This is a combinatorial characterization of what it means for the $U$-ultrapower of $\omega$ to be saturated in the sense of [10].

\section{THE THEOREMS}

We now introduce the central concept of this paper and state our main results.

Definition 1. We will say that selective (respectively semiselective, $P$-point, saturated) ultrafilters generically exist iff every filter of character $<c$ is included in a selective (respectively semiselective, $P$-point, saturated) ultrafilter. We will say that $Q$-points (respectively semi- $Q$-points) exist generically iff every filter of character $<d$ is included in a $Q$-point.

The choice of the term generic existence will be explained later. It should be clear that the generic existence of any type of ultrafilter implies that ultrafilters of that type do exist; that the generic existence of selective implies the generic existence of semiselective, $P$-point, and $Q$-point ultrafilters; and that the generic existence of $Q$-points implies the generic existence of semi- $Q$-points.

Theorem 2. The following are equivalent :

1. $m_{c}=c$,

2. Selective ultrafilters exist generically,

3. Semiselective ultrafilters exist generically. 
Theorem 3. The following are equivalent :

1. $m_{c}=d$,

2. Q-points exist generically,

3. Semi-Q-points exist generically.

Theorem 4 (Ketonen [11]). The following are equivalent :

1. $c=d$,

2. P-points exist generically.

Theorem 5 (Fremlin and Nyikos [16]). The following are equivalent :

1. $m_{c}=c=2^{<c}$,

2. Saturated ultrafilters exist generically,

3. Saturated ultrafilters exist.

In [16], Nyikos and Fremlin actually prove the equivalence of 1 and 3 in Theorem 5; they show that saturated ultrafilters exist iff $m_{c}=c=2^{<c}$. The beauty of this result is that it relates the existence of a special kind of ultrafilter to a simple combinatorial statement regarding the equality of certain cardinals. The latter statement makes no mention of ultrafilters, and its content may be understood by one who has no knowledge of, or interest in, ultrafilters. Upon learning of the Fremlin-Nyikos result, the current author sought to obtain similar results for other special ultrafilters: $P$-points, $Q$-points, and selectives. Upon reflection, it now appears that this program cannot succeed; all known proofs of the existence of these ultrafilters proceed by transfinite induction and actually establish the generic existence of the ultrafilter in question, since the construction may begin with any prescribed filter of the appropriate character. Indeed, the proof of the existence of saturated ultrafilters from the hypothesis $c=m_{c}=2^{<c}$ found in [16] can be easily modified to establish the generic existence of saturated ultrafilters, as noted in Theorem 5. (A proof that saturated ultrafilters exist generically in the Cohen model can be found in [8] and [17]. The only property of the Cohen model actually used in that construction is that it satisfies $c=m_{c}$ and $2^{<c}=c$.)

This prompted the current author to investigate whether or not the generic existence of the other special ultrafilters was equivalent to an equation about cardinals. Upon reflection, it was clear that [11] had already supplied the answer for the $P$-point case. There Ketonen established what we are now calling the generic existence of $P$-points under the hypothesis that $c=d$. He also showed that there exists a filter of character $d$ which is not included in a $P$-point, thus giving us the reverse implication for Theorem 4. (This point is discussed further in [9].) It remained for the author to supply the answer for the case of $Q$-points and selectives. These results were reported above.

The concept of generic existence also clarifies the relationship between semi$Q$-points and $Q$-points. While the existence of semi- $Q$-points appears to be weaker than that of $Q$-points, in the models where it is known that $Q$-points do not exist (models obtained by iteratively adding Laver or Mathias reals, studied by Miller in [14], or Shelah's model for Blass's Near Coherence of Filters 
principle, described in [6]), semi- $Q$-points do not exist either. Moreover, when Kunen showed in [12] that selective ultrafilters do not exist in the random real model, he actually proved that semiselectives are not found there either. One might conjecture that the existence of $Q$-points and selectives is equivalent to that of semiselectives and semi- $Q$-points respectively. While this remains open, we have that the generic existence of these types of ultrafilters are equivalent.

In the next section, we present the proofs of Theorems 2 and 3 . In each, the implication from 2 to 3 is trivial. Moreover the implications from 1 to 2 of both theorems are quite easy and essentially mimic well-known standard constructions. It is the converse which is new. However, we present both directions so as to provide a complete proof of our characterizations. We present these proofs in a series of short lemmas.

\section{THE PROOFS}

Lemma 6. Assume that $m_{c}=c$. Let $H$ be a filter-base whose cardinality is $<c$. Then there exists a selective ultrafilter $U$ which includes $H .[1 \rightarrow 2$ in Theorem 2.]

Proof. Let ${ }^{\omega} \omega=\left\{f_{\tau} \mid \tau \in c\right\}$. We construct a sequence of subsets of $\omega,\left\langle A_{\tau}\right| \tau \in$ $c\rangle$, such that $f_{\tau}$ is either one-to-one or bounded on $A_{\tau}$ and such that $H \cup$ $\left\{A_{\tau} \mid \tau \in c\right\}$ has the Finite Intersection Property (FIP). We may then taken $U$ to be any ultrafilter which includes this family; such a $U$ is necessarily selective.

The construction of the $A_{\tau}$ 's is done by transfinite recursion. At stage $\tau$, we assume for induction hypothesis that $H \cup\left\{A_{\sigma} \mid \sigma \in \tau\right\}$ has the FIP. We may then apply the following lemma, taking the $F$ of the lemma to be this family and the $f$ of the lemma to be our $f_{\tau}$.

Lemma 7. Assume that $m_{c}=c$. Let $F$ be a filter base of cardinality $<c$ and let $f: \omega \rightarrow \omega$. Then there exists a set $A$ such that $f$ is either one-to-one or bounded on $A$ and such that $F \cup\{A\}$ enjoys the FIP.

Proof. For each $k \in \omega$, either $\{n \mid f(n) \leq k\}$ meets each set in $F$ or $\{n \mid f(n)>$ $k$ \} meets each set in $F$.

Case 1. For some $k,\{n \mid f(n) \leq k\}$ meets each set in $F$. Take $A$ to be $\{n \mid f(n) \leq k\}$ and we are done.

Case 2. For every $k,\{n \mid f(n)>k\}$ meets each set in $F$. Let $P$ be the set of finite subsets of $\omega$ on which $f$ is one-to-one. Partially order $P$ by declaring a subset $p$ to be more informative than $q$ iff $p$ is an end-extension of $q$. For any $E \subseteq \omega$, let $D_{E}$ be the set of all $p \in P$ which have nonempty intersection with $E$. It follows from the case hypothesis that $D_{E}$ is dense in $P$ for every $E \in F$. There are fewer than $m_{c}=c$ sets in the filter $F$, so we may find a generic $G \subseteq P$ which meets $D_{E}$ for each $E \in F$. Then the set $A=\bigcup G$ is as desired. 
Lemma 8. Assume that $m_{c}=d$. Let $H$ be a filter-base of cardinality $<d$. Then there exists a Q-point ultrafilter $U$ which includes $H$. [1 $\rightarrow 2$ in Theorem 3.] Proof. Let $\left\{f_{\tau} \mid \tau \in d\right\}$ be a dominating family. We construct a sequence of subsets of $\omega,\left\langle A_{\tau} \mid \tau \in d\right\rangle$, such that $A_{\tau}$ is $f_{\tau}$-rare and such that $H \cup\left\{A_{\tau} \mid \tau \in d\right\}$ has the Finite Intersection Property (FIP). This family is then included in an ultrafilter $U$ which must necessarily be a $Q$-point.

The construction of the $A_{\tau}$ 's is again done by transfinite recursion. At stage $\tau$, we again assume for induction hypothesis that $H \cup\left\{A_{\sigma} \mid \sigma \in \tau\right\}$ has the FIP. We may then apply the following lemma, taking the $F$ of the lemma to be this family and the $f$ of the lemma to be our $f_{\tau}$.

Lernma 9. Assume that $m_{c}=d$. Let $F$ be a filter base of cardinality $<d$ and let $f: \omega \rightarrow \omega$. Then there exists an $f$-rare set $A$ which meets each $E$ in $F$.

Proof. Let $P$ be the set of finite subsets of $\omega$ which are $f$-rare. That is, if $p \in P$, and $i, j \in p$ with $i<j$, then $f(i)<j$. Partially order $P$ by declaring a condition $p$ to be more informative than $q$ iff $p$ is an end-extension of $q$. For any infinite subset $E$ of $\omega$, let $D_{E}$ be the set of all $p \in P$ which have nonempty intersection with $E . D_{E}$ is clearly dense (provided $E$ is infinite). There are fewer than $m_{c}=d$ sets in the filter $F$, so we may find a generic $G \subseteq P$ which meets $D_{E}$ for each $E \in F$. Then the set $A=\bigcup G$ is as desired.

Remark. It is easy to see that an ultrafilter cannot be generated by fewer than $m_{c}$ sets. Thus we may actually construct $2^{c}$ distinct selective ultrafilters from the hypothesis that $m_{c}=c$, as was done in the Cohen model in [8]. Similarly we may construct $2^{d}$ rapid ultrafilters from the hypothesis that $m_{c}=d$. However, the existence of $2^{c}$ selective ultrafilters is not equivalent to the generic existence of selectives. Indeed, Baumgartner has pointed out [2] that there is a model in which $m_{c}=d=\omega_{1}<c=\omega_{\omega_{1}}$ but which also contains $2^{c}$ selective ultrafilters. This model is due to Bell and Kunen [5] and is cited by Steprans in [19]. It is constructed via a finite-support c.c.c. iterated forcing of length $\omega_{1}$ in such a way that the intermediate models $V\left[G_{\alpha+1}\right]$ satisfy Martin's axiom and $c=\omega_{\alpha+2}$. In the final model $c=\omega_{\omega_{1}}$, but the presence of MA in the intermediate models allows us to carry out many MA constructions. In particular, in this model, $2^{c}$ selective ultrafilters may be constructed. In this model, $c$ is singular; it remains open whether the regularity of $c$ and the existence of $2^{c}$ selective ultrafilters implies that $m_{c}=c$.

Lemma 10. Assume that every filter whose character is $<c$ is included in a semiselective ultrafilter. Then $m_{c}=c .[3 \rightarrow 1$ in Theorem 3.]

Proof. Let $H$ be a subset of ${ }^{\omega} \omega$ of size $<c$. We seek a function $g$ which meets each $h \in H$. 
Partition $\omega$ into blocks of finite sets $B_{0}, B_{1}, B_{2}, \ldots, B_{n}$ so that $\left|B_{n}\right|=$ $n^{2}$. Partition each $B_{n}$ into $n$ subblocks $L_{n}^{1}, L_{n}^{2}, \ldots, L_{n}^{n}$ so that each $L_{n}^{k}$ has cardinality $n$. Let $X_{n}=\left\{p \mid p: B_{n} \rightarrow \omega\right\}$. Let $X=\bigcup\left\{X_{n} \mid n \in \omega\right\}$. $X$ is countable; it will be the index set for the filter and ultrafilter that we will construct.

Define $N: X \rightarrow \omega$ by setting $N(p)$ equal to the unique $n$ such that $p \in X_{n}$, so that $N^{-1}\{n\}=X_{n}$. Define $A_{h}$, for $h \in H$, and $F$ by:

$$
\begin{gathered}
A_{h}=\left\{p \in X \mid \forall k \in[1, N(p)] \exists b \in L_{N(p)}^{k} p(b)=h(b)\right\} \\
F=\left\{A_{h} \mid h \in H\right\} \cup\{\{p \mid N(p)>j\} \mid j \in \omega\}
\end{gathered}
$$

We check that $F$ has the FIP. Suppose we are given finitely many subsets $A_{h 1}, A_{h 2}, \ldots, A_{h n}$ and a set of the form $\{p \mid N(p)>j\}$ for $j \in \omega$. We may assume that $n>j$. For $k=1,2, \ldots, n$, write $L_{n}^{k}=\left\{b_{1}^{k}, b_{2}^{k}, \ldots, b_{n}^{k}\right\}$. Define $p: B_{n} \rightarrow \omega$ by $p\left(b_{1}^{k}\right)=h_{1}\left(b_{1}^{k}\right)$ for each $i$ and $k$ from $[1, n]$. Such a $p$ is in each $A_{h 1}$ as well as in $\{p \mid N(p)>j\}$, and thus witnesses the FIP for $F$.

Now apply the hypothesis to extend $F$ to a semiselective ultrafilter $U$. The function $N$ is not bounded on any set in $U$; hence we may find a set $E \in U$ such that each $E \cap X_{n}$ contains $n$ (not necessarily distinct) elements. For each $n \in \omega$ write $E \cap X_{n}=\left\{q_{n}^{1}, q_{n}^{2}, \ldots, q_{n}^{n}\right\}$ and define $p_{n}: B_{n} \rightarrow \omega$ so that $p_{n}(b)=q_{n}^{k}(b)$ whenever $b \in L_{n}^{k}$. Let $g=\bigcup\left\{p_{n}: n \in \omega\right\}$.

We conclude the proof by showing that for each $h \in H, g$ agrees with $h$ somewhere. For each $h, E \cap A_{h}$ is nonempty and must meet some $X_{n}$. For such $n$, find $k$ such that $q_{n}^{k} \in E \cap A_{h} \cap X_{n}$. As $q_{n}^{k} \in A_{h}$, we may find $b \in L_{n}^{k}$ such that $q_{n}^{k}(b)=h(b)$. However for each such $b, g(b)=p_{n}(b)=q_{n}^{k}(b)$; hence $g(b)=h(b)$ as required.

Lemma 11. Assume that every filter-base of cardinality $<d$ is included in $a$ semi-Q-point. Then $m_{c}=d .[3 \rightarrow 1$ in Theorem 3.]

Proof. This proof is similar to the previous one. We are given a family $H$ of functions which now has cardinality $<d$ and we seek a function $g$ which agrees with each $h \in H$ somewhere.

Let $B_{n}$ and $L_{n}^{k}$ be defined as in the previous proof. For each finite subset $S$ of $H$, define $f_{s}: \omega \rightarrow \omega$ via $f_{s}(n)=\operatorname{Max}\left\{h(i) \mid i \in B_{n}, h \in S\right\}$. Find a function $f$ which is not eventually dominated by any of the $f_{s}$ 's. $\left(\left\{n \mid f(n)>f_{s}(n)\right\}\right.$ is infinite.) Let $X_{n}=\left\{p \mid p: B_{n} \rightarrow[0, f(n)]\right\}$. Note that the $X_{n}$ 's are finite. Again we let $X$ be the union of the $X_{n}$ 's.

We define $N, A_{h}$, and $F$ exactly as before.

To check that $F$ has the FIP, we assume that we are given finitely many subsets $A_{h_{1}}, A_{h_{2}}, \ldots, A_{h_{m}}$ and a set of the form $\{p \mid N(p)>m\}$, and we seek $p$ which is in all of these sets. Let $S=\left\{A_{h_{0}}, A_{h_{1}}, \ldots, A_{h_{m}}\right\}$. Find $n$ sufficiently 
large that $n \geq m$ and $f(n)>f_{s}(n)$. As in the previous proof, write $L_{n}^{k}=$ $\left\{b_{1}^{k}, b_{2}^{k}, \ldots, b_{n}^{k}\right\}$. Define $p: B_{n} \rightarrow \omega$ such that $p\left(b_{1}^{k}\right)=h_{1}\left(b_{1}^{k}\right)$ whenever $i \leq m$ and $=0$ otherwise. Thus for each $b \in B_{n}, p(b) \leq f_{s}(n)<f(n)$; hence $p \in X_{n}$ and $p$ may be used for our FIP-witness.

Extend $F$ to a semi- $Q$-point $U$. The function $N$ is finite-to-one over $U$; this was the purpose of making the $X_{n}$ 's finite. Hence, we may again find a set $E \in U$ such that $\left|E \cap X_{n}\right| \leq n$ for each $n \in \omega$. We complete the proof by constructing, from this set $E$, a function $g$ that is as desired, just as in the previous proof.

\section{Closing REMARKS}

Genericity. We now mention another formulation of the results given above. For each cardinal $\lambda$ of uncountable cofinality, we let $Q_{\lambda}$ be the set of filters of character $<\lambda$. We partially order $Q_{c}$ by declaring a filter $F^{\prime}$ to be more informative than another filter $F$ iff $F \subseteq F^{\prime}$. Forcing with $Q_{\lambda}$ adjoints a generic ultrafilter $U$ (characterized by $F+A \in U$ iff $A \in F$ ) yet adds no new functions. As noted in [9], if $Q_{c}$-generic ultrafilters are selective, then selective ultrafilters exist in the ground model $V$. Moreover if $Q_{c}$-generic ultrafilters are $P$-points, then $P$-points exist in the ground model. If we unravel "All $Q_{c}$ generic ultrafilters are selective," we see that it is equivalent to the following statement, which is essentially the conclusion of Lemma 7:

$$
\begin{aligned}
& \forall F \in Q_{c}, \forall f: \omega \rightarrow \omega, \exists F^{\prime} \in Q_{c} f \text { is one-to-one } \\
& \text { or bounded on a set in } F^{\prime} \supseteq F .
\end{aligned}
$$

The proof given above reveals that this statement is equivalent to the generic existence of selectives. Indeed, this motivated the choice of the term generic existence; selective ultrafilters exist generically iff all $Q_{c}$-generic ultrafilters are selective. Similarly it is easy to see that $P$-point, semiselective, and saturated ultrafilters exist generically iff all $Q_{c}$-generic ultrafilters are respectively $P$-points, semiselectives, or saturated. Similarly $Q$-points and semi- $Q$-points exist generically iff all $Q_{d}$-generic ultrafilters are $Q$-points.

Booth's Lemma and $P_{c}$-points. The reader might wonder if there is a special type of ultrafilter whose generic existence is equivalent to the full strength of Martin's axiom. This appears not to be the case, since all ultrafilter constructions using Martin's axiom only use Martin's axiom applied to sigma-centered föcing, which Bell [4] has shown to be equivalent to the statement known as $P(c)$ of Booth's lemma. It is easy to prove that these statements are all equivalent to the generic existence of a $P_{c}$-point ultrafilter, as defined in [7].

Singularities. We conclude by noting that the generic existence of $P$-points, $Q$-points, and selectives is not equivalent to the mere existence of those ultrafilters. For example, in [3] Baumgartner and Laver have shown that in the model obtained by iteratively adding $\omega_{2}$ perfect reals to a model of $\mathrm{CH}$, there exist selective ultrafilters, each of which has character $\omega_{1}$. However, in this model 
$m_{c}=d=\omega_{1}<c$; so in this model we have neither selective ultrafilters nor even $P$-points existing generically. One might be tempted to say that the ultrafilters which exist in this model are singularities. This suggests the first of our

\section{OPEN PROBLEMS}

1. Can one find a characterization of singular $P$-points, $Q$-points, and selective ultrafilters, so that one could obtain results such as "If selective ultrafilters exist, then either they exist generically or there is a singular selective"?

2 . Can one prove that $Q$-points exist iff semi- $Q$-points exist, or that semiselectives exist iff selectives exist?

3. Under the assumption that $c$ is regular, is the existence of $2^{c}$ selective ultrafilters equivalent to the generic existence of selectives? Is the existence of $2^{d} Q$-points equivalent to the generic existence of $Q$-points?

\section{REFERENCES}

1. T. Bartoszynski, Combinatorial aspects of measure and category, Univ. Warszawski Inst. Mat., preprint 9/84, 1984.

2. J. Baumgartner and R. Laver, Iterated perfect set forcing, Ann. Math. Logic 17 (1979), 271-288.

3. J. Baumgartner, private letter to the author, 11 November 1988.

4. M. Bell, On the combinatorial principle $P(c)$, Fund. Math. 114 (1981), 149-157.

5. M. Bell and K. Kunen, On the PI character of ultrafilters, C. R. Math. Rep. Acad. Sci. Canada 3 (1981), 351-356.

6. A. Blass and S. Shelah, There may be simple $P_{\aleph_{1}}$ and $P_{\dot{\aleph}_{2}}$ points and the Rudin-Keisler order may be downward directed, Ann. Pure Appl. Logic 33 (1987), 213-243.

7. D. Booth, Ultrafilters on a countable set, Ann. Math. Logic 2 (1970-1), 1-24.

8. R. M. Canjar, Countable ultraproducts without CH, Ann. Pure Appl. Logic 37 (1988), 1-79.

9. __ Small-filter forcing, J. Symbolic Logic 51 (1986), 526-546.

10. C. C. Chang and H. J. Keisler, Model theory, North-Holland, 1973.

11. J. Ketonen, On the existence of P-points, Fund. Math. 92 (1976), 91-99.

12. K. Kunen, Some points in $\beta N$; Proc. Cambridge Philos. Soc. 78 (1980), 385-398.

13. A. Mathias, Remarks on rare filters, Infinite and Finite Sets, Colloq. Math. Soc. János Bolya, North-Holland, Amsterdam, 1975.

14. A. Miller, There are no Q-Points in Laver's model for the Borel conjecture, Proc. Amer. Math. Soc. 78 (1980), 103-106.

15. _ A characterization of the least cardinal for which the Baire category theorem fails, Proc. Amer. Math. Soc. 86 (1982), 498-502.

16. P. J. Nyikos and D. H. Fremlin, Saturating ultrafilters on $N$, J. Symbolic Logic 54 (1989), 708-718.

17. J. Roitman, Non-isomorphic H-fields from non-isomorophic ultrapowers, Math. Z. 181 (1982), 93-96.

18. S. Shelah, Proper forcing, Lecture Notes in Math., vol. 940, Springer-Verlag, 1982, pp. 213-221.

19. J. Steprans, Cardinal Arithmetic and $\aleph-B o r e l ~ s e t s$, Proc. Amer. Math. Soc. 84 (1982), 121126.

20. A. Taylor, On the existence of P-points and Q-points, preprint. 
21. E. van Douwen, The integers and topology, Handbook of Set-Theoretic Topology (K. Kunen and J. E. Vaughan, eds.), North-Holland, 1984.

22. W. Weiss, Versions of Martin's axiom, Handbook of Set-Theoretic Topology (K. Kunen and J. E. Vaughan, eds.), North-Holland, 1984.

Department of Computer Science, Mathematics, and Statistics, Yale Gordon College of Liberal ARTs, Baltimore, Maryland 21201 